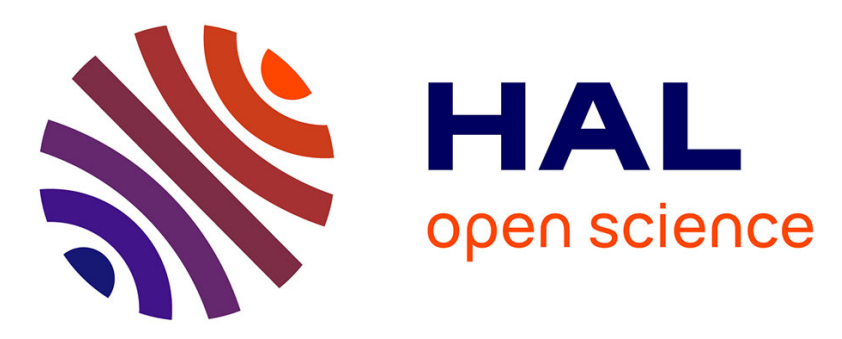

\title{
Analysis of blazed diffractive optical elements formed with artificial dielectrics
}

Céline Ribot, Philippe Lalanne, Mane-Si Laure Lee, Brigitte Loiseaux, Jean-Pierre Huignard

\section{To cite this version:}

Céline Ribot, Philippe Lalanne, Mane-Si Laure Lee, Brigitte Loiseaux, Jean-Pierre Huignard. Analysis of blazed diffractive optical elements formed with artificial dielectrics. Journal of the Optical Society of America. A Optics, Image Science, and Vision, 2007, 24 (12), pp.3819-3826. hal-00846701

\section{HAL Id: hal-00846701 \\ https://hal-iogs.archives-ouvertes.fr/hal-00846701}

Submitted on 19 Jul 2013

HAL is a multi-disciplinary open access archive for the deposit and dissemination of scientific research documents, whether they are published or not. The documents may come from teaching and research institutions in France or abroad, or from public or private research centers.
L'archive ouverte pluridisciplinaire HAL, est destinée au dépôt et à la diffusion de documents scientifiques de niveau recherche, publiés ou non, émanant des établissements d'enseignement et de recherche français ou étrangers, des laboratoires publics ou privés. 


\title{
Analysis of blazed diffractive optical elements formed with artificial dielectrics
}

\author{
Céline Ribot, ${ }^{1,2}$ Philippe Lalanne, ${ }^{1}$ Mane-Si-Laure Lee, ${ }^{2, *}$ Brigitte Loiseaux, ${ }^{2}$ and Jean-Pierre Huignard ${ }^{2}$ \\ ${ }^{1}$ Laboratoire Charles Fabry de l'Institut d'Optique, CNRS, Université Paris-Sud, Campus Polytechnique, \\ RD 128, F-91127 Palaiseau Cedex, France \\ ${ }^{2}$ Thales Research \& Technology, RD 128, F-91767 Palaiseau Cedex, France \\ *Corresponding author: mane-si-laure.lee@thalesgroup.com
}

Received July 16, 2007; accepted September 7, 2007;

posted October 12, 2007 (Doc. ID 84844); published November 28, 2007

\begin{abstract}
A new hybrid method for the analysis of diffractive optical elements, which combines fully vectorial and scalar theories, is presented. It is suitable for use with elements of arbitrary large zone, even when the local feature size is of the order of the wavelength. To assess its applicability, we have performed cross-checking tests. The model is shown to accurately predict many optical properties of diffractive optical elements based on twodimensional artificial dielectrics, like the useful energy diffracted into the order of interest or the deterministic loss into high diffraction orders for an illumination with a wavelength different from the design wavelength or for highly oblique incidence. (C) 2007 Optical Society of America OCIS codes: $050.1380,050.1970,050.1950,230.1950$.
\end{abstract}

\section{INTRODUCTION}

Many theoretical tools are available for the analysis and the design of diffractive optical elements (DOEs). The most accurate description of diffraction can be achieved with fully vectorial computation methods. Solving Maxwell's equations requires a large amount of computing time, which limits the usefulness of fully vectorial methods with respect to design algorithms based on iterative techniques. Therefore approximations have to be incorporated. One of the most practical approaches makes use of Kirchhoff's approximation [1]. Within this approach, one assumes that the DOEs can be treated as infinitely thin diffraction screens that modulate the transverse scalar amplitude distribution of the incident wave field. Absorbing elements are then described as amplitude modulations of the incident wave field, refractive index distributions are described as phase-only modulations, and the phase delay is obtained by classical ray-tracing methods. For many applications, such as échelette-type elements or binary elements with local periodicities much larger than the wavelength of the incident wave, this approach and further extensions to incorporate finite-thickness effects $[1,2]$ or other refinements [3,4] provide sufficient accuracy.

One of the greatest problems in the analysis of DOEs arises when one combines large Fresnel zones and small structural features. However, developments in microlithography and associated technologies enable the manufacture of large-area DOEs that are locally composed of tiny subwavelength features, providing interesting potentialities. On one side, one finds DOEs that are locally optimized through fully vector theory treatments. This approach has been proved fruitful [5,6] for applications relying on plane wave illuminations at a single wavelength and at a given incidence angle. On the other side, one finds a variety of components with operations that rely on the analogy between subwavelength binary features and artificial media. Within this approach, new properties or performances that cannot be achieved with conventional échelette DOEs become accessible to the optical designer. For instance, highly efficient blazing occurs in a broadband spectrum $[7,8]$ or in a broad range of incidence angles, even for DOEs with zone widths of only a few wavelengths [9]. The efficiency of the scalar-domain limit may be broken [10-12]. Recent examples of components relying on the artificial-medium analogy can be found in [13-16].

The fabrication constraints require a characteristic size comparable with the incident wavelength for the current smallest features. Since homogenization theories do not strictly apply, the design and analysis must combine scalar and vector theories. The purpose of the present work is to derive a simple tool that permits the analysis of DOEs made of large Fresnel zones, each composed of thin subwavelength structural details. We show that these elements can be fully analyzed by simple local vector theory calculations and that many important effects can be quantitatively predicted, like the impact of oblique illumination, the distribution of the light scattered into the spurious diffracted orders, and the effect of the illumination wavelength, especially when it differs from the nominal one. In Section 2, the hybrid model so-called unwrappedphase-extension method (UPEM) is presented. It relies on the unwrapped phase functions associated with the DOE, contrary to previous works based on a spatial sampling [17]. The phase expansion allows for an intuitive interpretation of the intricate pattern generated by the superposition of all diffracted orders. Actually, within this representation, the transmittance is shown as a coherent superposition of scalar waves, the different orders of the DOE, with weighted amplitudes that are proportional to the square root of the diffraction efficiencies and that are 
calculated with fully vectorial tools. Section 3 describes numerical aspects relative to the calculation of the efficiency into all diffracted orders, and especially high orders. In Section 4, we apply the model to several DOEs that have been recently proposed in the literature. The performance of the model is illustrated by fully characterizing the optical properties of the scattered waves. Section 5 summarizes the contribution.

\section{HYBRID MODEL}

In the design of modern optical systems, DOEs are incorporated to correct both chromatic and field aberrations. At the initial stage of the design, DOE profiles are calculated so as to implement a target unwrapped phase function $\psi(x, y)$, where $x$ and $y$ are the coordinates of a point in the planar Cartesian system associated with the diffractive element. The phase $\psi(x, y)$ will play a key role in the following analysis. It may be arbitrary. For instance, $\psi(x, y)$ may exhibit an intricate dependence on the space variables, as is the case for kinoforms [18]. For hybrid lens doublets defined for chromatic-aberration correction, $\psi(x, y)$ includes the spherical phase of a slow lens with a focal length much larger than that of the doublet and eventual phase corrections for other geometric aberrations.

The model relies on two assumptions. First, it assumes that the unwrapped phase function is slowly varying at the wavelength scale. This implies that the model cannot take into account shadowing effects [19] that prevent the realization of $100 \%$ efficiencies. But for components with a characteristic size larger than $\approx 10 \lambda$, the model predictions remain accurate. The second assumption concerns the design of the DOE. We assume that, wherever the unwrapped phase function is equal to a given value $\psi$ modulo $2 \pi$, the DOE locally presents the same geometry, denoted $g$ hereafter. In other words, we assume that it is possible to define a $2 \pi$-periodic calibration function $\mathrm{F}$ so that

$$
\mathrm{F}: \psi \rightarrow g=\mathrm{F}(\psi)(\equiv 2 \pi)
$$

as illustrated in Fig. 1(a). The choice of the local geometry $g$, or equivalently of the function $\mathrm{F}$, is the art of the design and encompasses many different situations. In classical
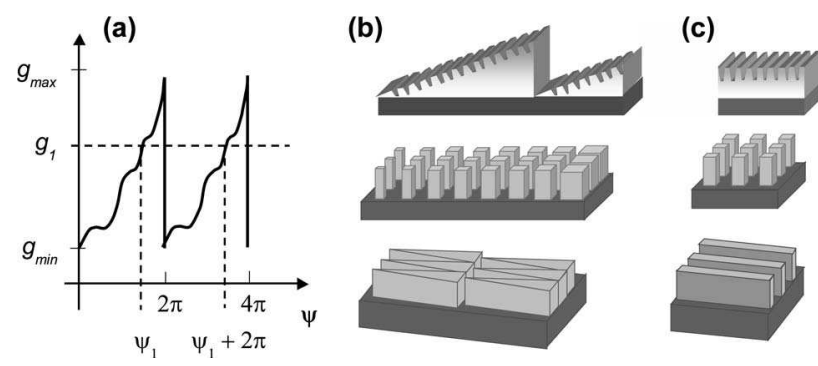

Fig. 1. Different calibration functions, $\mathrm{F}: g=\mathrm{F}(\psi)$, corresponding to different geometries $g$. (a) Sketch of the $2 \pi$ periodicity of $F$. (b) Examples of DOEs that can be analyzed with the model. From top to bottom: échelette-type DOEs replicated with their motheye-type antireflection coating [20], blazed-binary element, and blazed area-coded effective-medium structure [15]. (c) Corresponding local geometries $g$. designs relying on continuous échelette-type profiles or staircase multilevel binary profiles, the local geometry consists of a thin layer with a given thickness. However, it can be also more complex patterns either in metallic or dielectric material, like subwavelength grooves [21], ridges [22,23], holes [24], micropillars [10], or pie slices [15], as illustrated in Figs. 1(b) and 1(c). Although it precludes the analysis of fast lenses, the first hypothesis is not very restrictive. For instance, slow lenses or hybrid lenses used for aberration correction can be analysed. The second hypothesis does not represent a restriction. It is classical, in fact, since all DOEs fabricated so far rely on a periodic calibration function to our knowledge.

For the sake of simplicity, we only consider the DOE response in transmission, the response in reflection being obtained in a similar way. As any incident wave field can be expanded as a superposition of plane waves, it is sufficient to study the optical response of the DOE for a single plane wave. The angles $\theta$ and $\delta$ are arbitrary (Fig. 2 ). We denote by $\mathbf{W}_{i}$ a $6 \times 1$ vector formed by the electric and magnetic field vectors of the incident plane wave, and by $\mathbf{k}_{i}$ its wave vector. Because the unwrapped phase function is assumed to vary slowly at the wavelength scale, the wave field transmitted through the DOE is weakly modulated in amplitude and in phase. It is a quasi plane wave $\mathbf{W}_{t}$, whose wave vector $\mathbf{k}_{t}$ is related to $\mathbf{k}_{i}$ by Snell's law.

The incident electromagnetic field can be written as a superposition of two linearly polarized plane waves $\mathbf{W}_{i}$ $=\alpha \mathbf{W}_{\mathrm{TE}, i}+\beta \mathbf{W}_{\mathrm{TM}, i}$, where $\alpha$ and $\beta$ are two given complex coefficients. $\mathbf{W}_{\mathrm{TE}, i}$ is a TE-polarized (electric field vector perpendicular to the plane of incidence) plane wave with a unitary Poynting vector $z$ component. Similarly, $\mathbf{W}_{\mathrm{TM}, i}$ denotes the corresponding TM-polarized (electric field vector parallel to the plane of incidence) plane wave. The transmitted wave field $\mathbf{W}_{t}$ can also be written as a superposition of two linearly-polarized plane waves, $\mathbf{W}_{t}$ $=\nu \mathbf{W}_{\mathrm{TE}, t}+\mu \mathbf{W}_{\mathrm{TM}, t}$, where $\nu$ and $\mu$ are two given complex

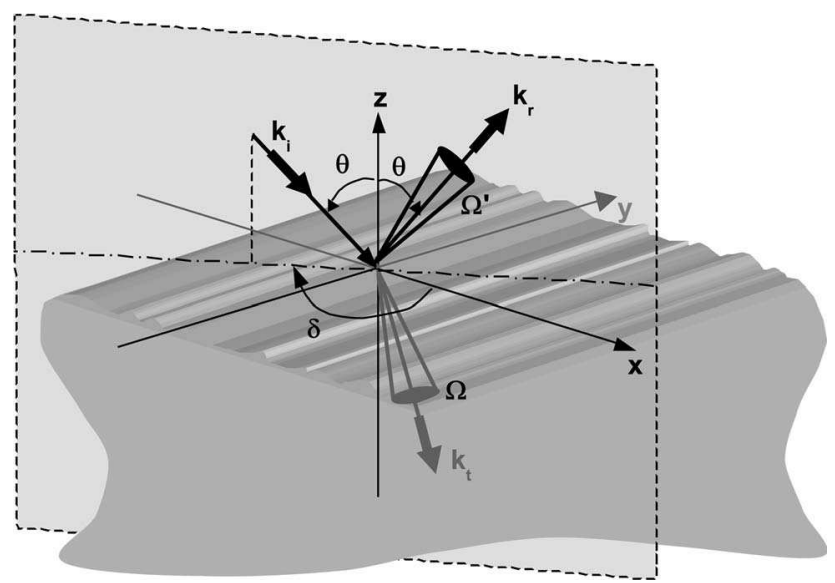

Fig. 2. Scattering of a DOE with a slowly varying unwrapped phase function. The DOE is illuminated by an unpolarized plane wave incident from medium 1 at arbitrary azimuthal $(\delta)$ and incidence $(\theta)$ angles. The transmitted wave field is weakly divergent and is contained in a small solid angle $\Omega$ centered around the wave vector $\mathbf{k}_{t}$ of the weakly modulated plane wave propagating in medium 2. Similarly, the reflected wave field is contained in a small solid angle $\Omega^{\prime}$ centred around the wave vector $\mathbf{k}_{r}$. 
coefficients and $\mathbf{W}_{\mathrm{TE}, t}\left(\mathbf{W}_{\mathrm{TM}, t}\right)$ represents a TE (TM) plane wave with a unitary Poynting vector $z$ component. Because Maxwell's equations are linear, the expansion coefficients $(\alpha, \beta)$ and $(\nu, \mu)$ on both sides of the DOE are linked by a Jones-type matrix:

$$
\left(\begin{array}{l}
\nu \\
\mu
\end{array}\right)=\mathbf{T}(x, y)\left(\begin{array}{l}
\alpha \\
\beta
\end{array}\right) .
$$

The matrix $\mathbf{T}(x, y)$ fully characterizes the DOE and defines the local complex transmittance of the DOE at every $(x, y)$ point of the component. The matrix $\mathbf{T}(x, y)$ provides a spatial representation that can be directly used to analyze the light scattered by the DOE, as it is the case for the so-called field-stitching approach [18], for instance. In contrast to direct sampling approaches in real space, which suffer [18] from the lack of a sound definition for the size of the local area to be considered for calculating the local $\mathbf{T}(x, y)$ matrices, we examine the scattering process in the phase space. This allows arbitrary $2 \mathrm{D}$ phase functions to be handled easily. Introducing the unwrapped phase function, Eq. (2) can be rewritten as

$$
\left(\begin{array}{l}
\nu \\
\mu
\end{array}\right)=\mathbf{T}(\psi)\left(\begin{array}{l}
\alpha \\
\beta
\end{array}\right)=\left(\begin{array}{ll}
t_{11}(\psi) & t_{12}(\psi) \\
t_{21}(\psi) & t_{22}(\psi)
\end{array}\right)\left(\begin{array}{l}
\alpha \\
\beta
\end{array}\right),
$$

where $\mathbf{T}(\psi)$ is now the transmittance Jones' matrix associated with the geometry $g=\mathrm{F}(\psi)$. In general, the set of matrices $\mathbf{T}(\psi)$ depends on the wavelength $\lambda$ of the incident plane wave and on the angles $\theta$ and $\delta$, but we omit making these dependences explicit for the sake of notational simplicity. First, according to the first hypothesis, the geometry can be locally seen as a periodic structure, see Fig. 1(c), and the $\mathbf{T}(\psi)$ matrix can be computed with a grating solver. More details will be provided in Section 3.

Then, as the DOE design uses the same local geometry wherever the unwrapped phase function is equal to a given value $\psi$ modulo $2 \pi$, the $\mathbf{T}(\psi)$ matrix is also $2 \pi$ periodic. Thus it can be expanded into a Fourier series

$$
\mathbf{T}(\psi)=\sum_{m=-\infty}^{m=\infty} \mathbf{C}^{m} \exp (j m \psi),
$$

with

$$
\mathbf{C}^{m}=\left(\begin{array}{ll}
c_{11}^{m} & c_{12}^{m} \\
c_{21}^{m} & c_{22}^{m}
\end{array}\right)=\frac{1}{2 \pi} \int_{0}^{2 \pi} \mathbf{T}(\psi) \exp (-j m \psi) \mathrm{d} \psi .
$$

According to Eq. (4), the transmitted wave field is seen as a superposition of elementary wave fields, the $\exp (j m \psi)$, weighted by the $\mathbf{C}^{m}$ matrix coefficients. Each wave field represents a spurious diffraction order [25] that is fully specified by the known unwrapped phase function $\psi(x, y)$. Considering, for instance, periodic DOEs, like Damman gratings [26], the elementary wave fields consist of plane waves. For hybrid refractive-diffractive lenses designed for chromatic-aberration compensation, the wave fields are out-of-focus spherical waves.

The $\mathbf{C}^{m}$ matrix coefficients, which take into account all polarization effects, are related to the diffraction efficiencies of the different orders. Because of our specific choice for the energy normalization of the linearly polarized plane waves $\mathbf{W}_{\mathrm{TE}, i}, \mathbf{W}_{\mathrm{TM}, i}$, the diffraction efficiencies, $\eta_{m}^{\mathrm{TE}}$ and $\eta_{m}^{\mathrm{TM}}$, into the $m$ th diffracted order, are simply given by

$$
\begin{aligned}
& \eta_{m}^{\mathrm{TE}}=\left(\left|c_{21}^{m}\right|^{2}+\left|c_{11}^{m}\right|^{2}\right), \\
& \eta_{m}^{\mathrm{TM}}=\left(\left|c_{22}^{m}\right|^{2}+\left|c_{12}^{m}\right|^{2}\right)
\end{aligned}
$$

for an incident plane wave with TE and TM polarizations, respectively. For unpolarized light, the efficiency $\eta_{m}$ into the $m$ th diffracted order is $\eta_{m}=1 / 2\left(\eta_{m}^{\mathrm{TE}}+\eta_{m}^{\mathrm{TM}}\right)$. These efficiencies are absolute efficiencies that include intrinsic Fresnel reflection losses at the DOE interface.

In practice, it might be useful to know relative efficiencies (normalized to the total energy diffracted in all transmitted orders) to finely predict the impact of these losses. To avoid fastidious calculations of the efficiencies into all diffracted orders, we use the Parseval relation, and the total energy diffracted in all transmitted orders is expressed as

$$
\sum_{m} \eta_{m}^{\mathrm{TE}}=\frac{1}{2 \pi} \int_{0}^{2 \pi}\left(\left|t_{21}(\psi)\right|^{2}+\left|t_{11}(\psi)\right|^{2}\right) \mathrm{d} \psi
$$

for a TE-polarized incident wave. A similar expression is straightforwardly obtained for TM polarization. Equation (8) allows us to get rid of the rapidly oscillating exponential terms encountered in the expressions of $\eta_{m}^{\mathrm{TE}}$ and $\eta_{m}^{\mathrm{TM}}$ for large $m$ values.

In general, DOEs are designed to be blazed into a specific order (often the first one), at a given wavelength and at a given angle of incidence. In principle, $100 \%$ of the incident energy should be diffracted into this order, and $\eta_{1}$ is equal to 1 . When the DOEs are illuminated at another wavelength or at another incidence, the first-order efficiency drops $\left(\eta_{1} \neq 1\right)$. The efficiencies $\eta_{m}(m \neq 1)$ into the other diffraction orders then carry some energy. This inevitable and deterministic loss contributes to the formation of background noise in the image plane, which degrades the image quality and affects the DOE optical transfer function [27]. We believe that the Fourier series expansion of Eq. (4) is an intuitive and meaningful representation of the background noise, especially when only the few highest-efficiency orders are considered. This quantitative approach cannot be obtained by other models relying on space expansions, as in Eq. (2).

\section{COMPUTATION OF THE DIFFRACTION EFFICIENCIES}

In this section we describe several aspects relative to the technical implementation of the model. For the sake of illustration, a test structure is examined. We consider a relatively intricate geometry, a blazed-binary DOE composed by the association of subwavelength holes and pillars, each defined on a sampling period $\Lambda_{s}$; see Fig. 3(a). In this design, the hole diameters and pillar sizes are chosen to implement a monotonic variation of the phase delay between 0 and $2 \pi$. The variation provides a nearly $100 \%$ first-order diffraction efficiency at the nominal 

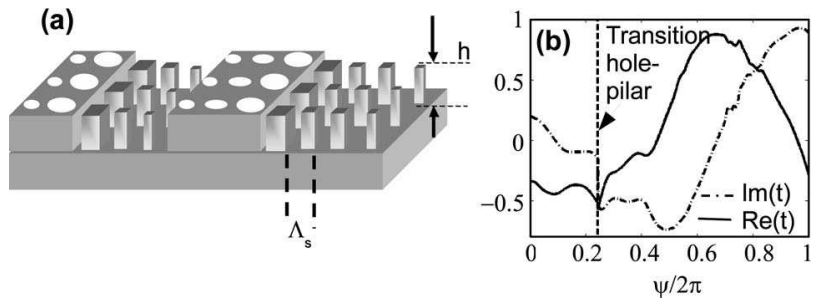

Fig. 3. (a) DOE composed of tiny pillars and holes etched into a $\mathrm{Si}_{3} \mathrm{~N}_{4}$ substrate $(n=2.1)$ for a nominal operation at $\lambda_{0}=0.8 \mu \mathrm{m}$. The sampling period is $\Lambda_{s}=0.5 \lambda_{0}$, and the etching depth is $h$ $=1.9 \lambda_{0}$. (b) Real (solid curve) and imaginary (dashed-dotted curve) parts of the transmitted zero-order coefficient $t=t_{11}=t_{22}$ as a function of the unwrapped phase $\psi$. The vertical dashed line shows the transition between the pillars $(\psi<0.25 \pi)$ and the holes $(\psi>0.25 \pi)$. The calculation has been performed for a normally incident plane wave at $\lambda=0.62 \lambda_{0}$.

wavelength $\lambda_{0}$. More details concerning the design and the beneficial impact of combining two different subwavelength geometries for achieving broadband blazing can be found in [8].

The optical properties of the DOE are fully characterized by the Fourier series expansion of Eq. (4). This expansion requires the calculation of single variableintegrals of the form

$$
c_{i, j}^{m}=\frac{1}{2 \pi} \int_{0}^{2 \pi} t_{i, j}(\psi) \exp (-j m \psi) \mathrm{d} \psi
$$

For the numerical calculation of the $c_{i, j}^{m}$ coefficients in Eq. (9), we use a two-step procedure.

The first step consists in computing the local complex transmittance coefficients $t_{i, j}(\psi)$ for all $\psi$ in the interval $[0 ; 2 \pi]$. This calculation is performed with fully vector theory software [28] relying on the rigorous coupled-wave analysis [29] and on its further improved versions [30-32]. It consists mainly in computing the transmitted zero-order amplitude coefficients of a $2 \mathrm{D}$ grating composed of artificial dielectric materials with a periodicity of the order of the wavelength. This vector theory step does not raise any difficulty, and we estimate that it could be performed with sufficient accuracy. Figure 3(b) shows the imaginary and real parts of $t(\psi)=t_{11}(\psi)=t_{22}(\psi)$ for a normally incident illumination at $\lambda=0.62 \lambda_{0}$. For this wavelength that strongly differs from the nominal one and that is close to the separation distance $\Lambda_{s}$, the component is no longer blazed, and the $t_{i, j}$ 's are discontinuous at the transition $(\psi=0.25 \pi)$ between the pillar and hole zones.

Once the functions $t_{i, j}(\psi)$ are known for all $\psi$, the second step of the analysis consists in computing the integral in Eq. (9). Numerical problems are anticipated for large $m$ values because of the presence of a rapidly oscillating exponential term in the integral. To fully overcome these problems, we perform the integration analytically. First the $[0 ; 2 \pi]$ interval is decomposed into a sequence of $M$ subintervals, denoted $I_{p}, I_{p}=\left[\psi_{p-1} ; \psi_{p}\right]$. In each subinterval $I_{p}, t_{i, j}(\psi)$ is locally approximated by a second-order polynomial $P_{p}(\psi)=a_{p} \psi^{2}+b_{p} \psi+c_{p}$, where the coefficients $a_{p}, b_{p}$, and $c_{p}$ are obtained from the $t_{i, j}(\psi)$ values at three points, $\psi_{p-1},\left(\psi_{p-1+} \psi_{p}\right) / 2$, and $\psi_{p}$. After an analytical integration step, we easily obtain the discrete summation

$$
\begin{aligned}
\int_{0}^{2 \pi} t_{i, j}(\psi) \exp (\kappa \psi) \mathrm{d} \psi= & \sum_{p=1}^{M} G_{p}\left(\psi_{p}\right) \exp \left(\kappa \psi_{p}\right) \\
& -G_{p}\left(\psi_{p-1}\right) \exp \left(\kappa \psi_{p-1}\right),
\end{aligned}
$$

where

$$
\begin{aligned}
G_{p}(\psi)= & \left(a_{p} / \kappa\right)^{2} \psi^{2}+\left(b_{p} / \kappa-2 a_{p} / \kappa^{2}\right) \psi+\left(2 a_{p} / \kappa^{3}-b_{p} / \kappa^{2}\right. \\
& \left.+c_{p} / \kappa\right) .
\end{aligned}
$$

The discrete summation in Eq. (10) is straightforwardly calculated. In Fig. 4, we illustrate the accuracy of the approach for the DOE of Fig. 3 and for a normal incidence plane wave illuminating the element from the substrate at $\lambda=0.62 \lambda_{0}$. We first calculate the diffraction efficiencies $\eta_{m}$ of the $N$ first diffracted orders, from $m=-500$ to $m$ $=500$, using Eqs. (10) and (11). The thin curves in Figs. 4(a) and 4(b) represent the total energy diffracted into the $N$ first orders, $\eta^{(N)}=\Sigma_{m=-N}^{N} \eta_{m}$, for the reflected and transmitted light, respectively, as a function of $N$. Then, we compare these calculated results with those obtained for the total reflected and transmitted efficiencies, $R$ and $T$, calculated by using the Parseval relation of Eq. (8). The total efficiency values, $R \approx 0.190$ and $T \approx 0.525$, are determined with an excellent accuracy by using the Simpson integration method [33] for 600 sampling phase points. As $N$ increases, the difference between the $\eta^{(N)}$ and the values obtained with the Parseval relation (horizontal bold lines) vanishes. For $N=500$, the deviation is smaller than $4 \times 10^{-5}$ for both plots. This demonstrates the accuracy of the analytical integration approach, even for computing diffracted energies of orders as high as 500 .

We have also performed many other direct tests of the model showing its effectiveness and accuracy. For instance, we have considered échelette-type DOEs, for which closed-form expressions are available for the diffraction efficiencies into arbitrary orders under normal incidence [25]. The agreement between the model predictions and the closed-form expressions was excellent in all studied cases: the difference did not exceed $10^{-6}$ for five wavelengths chosen in the interval $0.5 \lambda_{0}<\lambda<2 \lambda_{0}$ and for the 500 first reflected and transmitted orders. Additionally, we have performed tests for 1D blazed-binary gratings illuminated at $\lambda_{0}$ under TM and TE polarizations, for
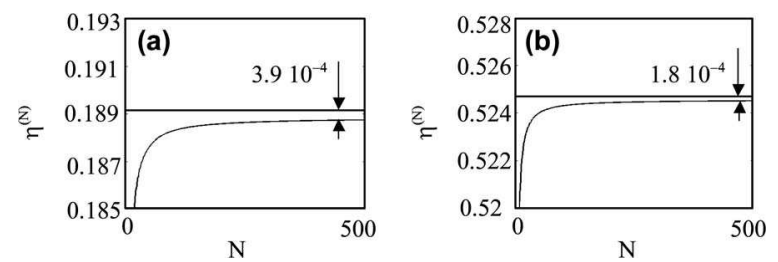

Fig. 4. Illustration of the accuracy achieved by the numerical technique developed to calculate the diffraction efficiencies into high orders. The bold horizontal lines, calculated with the Parseval relation, represent the total energy diffracted into all orders. The thin-solid curves, computed with Eqs. (10) and (11), represent the energy $\eta^{(N)}$ diffracted into the $N$ first orders, from $-N$ to $+N$. (a) Reflectance. (b) Transmittance. All results are obtained for the pillar-hole geometry of Fig. 3 at $\lambda=0.62 \lambda_{0}$. 
which the 20 first-order diffraction efficiencies of gratings with periods as large as $50 \lambda$ can be accurately calculated with the rigorous coupled-wave analysis. Again quantitative agreement between the model predictions and the rigorous coupled-wave analysis computational data has been achieved; the deviation $(<0.005)$ is comparable with the estimated uncertainty of the vector theory data.

All these tests show that the model is able to provide highly accurate data for the reflected and transmitted diffraction efficiencies, even into high-order background wave fields.

\section{APPLICATIONS}

In this Section, we apply the UPEM to the analysis of several types of DOE composed of subwavelength patterns in a dielectric material. Provided that the subwavelength patterns are small enough compared with the wavelength of the incident light, they are not resolved by the light (in the sense of the far-field diffraction). Thus the heterogeneous structure behaves as an artificial material with an effective refractive index. By controlling the local fraction of etched material, it is possible to implement a gradual variation of the effective index, and in turn to design DOEs with an efficient blazing. Because the electromagnetic response of the subwavelength patterns strongly depends on the incident wavelength (the artificial dielectric is dispersive) and on the incidence angle, the analysis cannot be performed with scalar theories only, and hybrid models like the present one are required.

\section{A. Optical Performance Analysis of Blazed-Binary Diffractive Optical Elements}

In this subsection, we show that the UPEM may be used for fully analyzing the optical properties of blazed-binary DOEs, including the prediction of the background-order efficiencies at oblique incidence. For the sake of illustration, we consider the DOE previously considered in Fig. 3. We emphasize that the optical properties of this type of DOE cannot be analyzed with fully vectorial theories, for zone widths larger than a few wavelengths. This is especially stringent for the general case of oblique incidence, for which no symmetry can be used to lower the computational loads. With the UPEM, since it has to be performed only for elementary subwavelength geometries, the 3D fully vectorial treatment can be achieved at low computational loads.

Figure 5 summarizes the main optical properties of the blazed-binary DOE composed of subwavelength holes and pillars. All curves are normalized to the total energy diffracted into all transmitted orders, and all results hold for an illumination incident from the substrate. Details concerning the geometrical parameters (hole diameters, pillar size...) and the design at the blaze wavelength can be found in the caption of Fig. 3.

The wavelength dependence of the first-order efficiency $\eta_{1}$ as a function of the normalized wavelength $\lambda / \lambda_{0}$ is shown in Fig. 5(a). For the calculation, we have assumed a normal incidence and a nondispersive material ( $n$ $=2.1$ ), but the material properties can be easily incorporated into the model, since all computations are per-
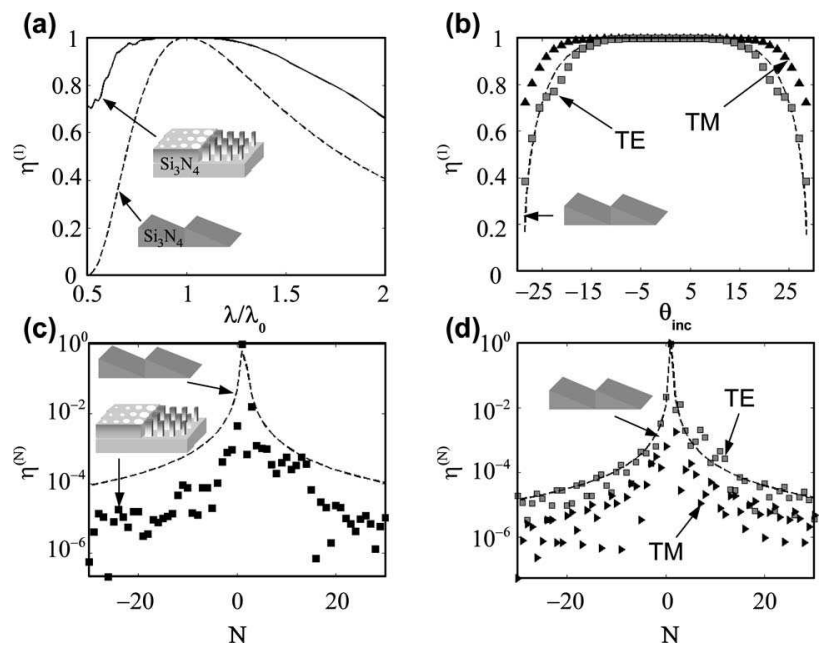

Fig. 5. Optical properties of blazed-binary DOE with subwavelength holes and square pillars etched in a $\mathrm{Si}_{3} \mathrm{~N}_{4}$ substrate $(n$ =2.1), as compared with échelette-type DOEs (dashed curves). (a) Wavelength dependence of the first-order diffraction efficiency (solid curve) for normal incidence and for unpolarized light. (b) First-order diffraction efficiency as a function of the incidence angle $\theta$ in $\mathrm{Si}_{3} \mathrm{~N}_{4}$ for a null azimuthal angle $(\delta=0)$ at $\lambda=\lambda_{0}$. Squares, TE polarization, triangles, TM polarization. (c), (d) Energy scattered into the background orders for normal incidence and $\lambda \approx 0.584 \lambda_{0}$ (c) and for oblique incidence $\left(\theta=18^{\circ}\right.$ in the substrate) and $\lambda=\lambda_{0}$ (d). All curves are normalized to the total energy diffracted in all transmitted orders, and all results hold for an incident illumination impinging on the DOE from the substrate side.

formed in the spectral domain. A remarkable blazing effect over more than one octave $\left(\eta_{1}>0.95\right)$ is predicted. For the sake of comparison, we also show the first-order diffraction efficiency (dashed curve) of an échelette-type DOE in $\mathrm{Si}_{3} \mathrm{~N}_{4}$ designed for blazing operation into its first order at $\lambda_{0}$. For these types of element, closed-form expressions are available for the efficiencies $\eta_{m}$ into all orders in the limit of infinitely large échelettes and for normal incidence, and $\eta_{m}$ is given by [26]

$$
\eta_{m}=\operatorname{sinc}^{2}\left(\lambda_{0} / \lambda-m\right)
$$

where $\operatorname{sinc}(x)=\sin (\pi x) /(\pi x)$.

Figure 5(b) shows the first-order diffraction efficiency as a function of the incidence angle $\theta_{\text {inc }}$ (in the $\mathrm{Si}_{3} \mathrm{~N}_{4}$ substrate) for $\lambda=\lambda_{0}$. Note that $\theta_{\text {inc }}=28.4^{\circ}$ corresponds to $90^{\circ}$ in air. For both polarizations, a large plateau $\left(-10^{\circ}<\theta\right.$ $<10^{\circ}$ ) with nearly $100 \%$ blazing is obtained. It is slightly larger than the plateau predicted for échelette-type DOEs with the model. We notice that $\eta_{1}$ weakly depends on the polarization of the incident light, the deviation being smaller than 0.02 for $|\theta|<13^{\circ}$. We have also performed similar computations for oblique incidence with a $45^{\circ}$ azimuthal angle. Almost identical results were obtained, the difference being smaller than 0.02 for $|\theta|<15^{\circ}$. As expected, the use of centrosymmetric subwavelength patterns (circular holes and square pillars) guarantees that the DOE response is weakly sensitive to the polarization state.

In Figs. 5(c) and 5(d), we show the diffraction efficiencies into the background orders. Figure 5(c) is obtained 
for normal incidence at $\lambda=0.584 \lambda_{0}$, and Fig. 5(d) for $\theta$ $=18^{\circ}\left(18^{\circ}\right.$ in the medium corresponding to $40^{\circ}$ in air) at $\lambda=\lambda_{0}$. For the sake of comparison, we have also plotted the efficiencies for échelette DOEs with the dashed curve. These efficiencies are obtained with Eq. (12) for the results shown in Fig. 5(c) and with the model for the results shown in Fig. 5(d). It is obvious that the first-order efficiency of the blazed-binary DOE is much larger than the background-order efficiencies, which scale as $(1 / N)^{2}$ for large $N$. Even for wavelengths largely different from the nominal one and even for large incidence angles, the firstorder efficiency remains almost 2 orders of magnitude larger than that of the highest background-order efficiency (usually the zero or second order).

Overall, Fig. 5 shows the remarkable performance of the blazed-binary DOEs with subwavelength pillars and holes, in comparison with that achieved with a classical échelette profile. Such results clearly illustrate the UPEM's capacity to quantitatively give access to many important properties of blazed-binary DOEs with unwrapped phase functions that are slowly varying at the wavelength scale. We are currently pursuing the fabrication of such elements for thermal-imaging applications.

\section{B. Optical Analysis of One-Dimensional Blazed-Binary and Area-Coded Effective Medium Diffractive Optical Elements}

As shown in the previous example, the optical properties of DOEs, with unwrapped phase functions slowly varying at the wavelength scale, are intrinsically governed by the subwavelength patterns used for encoding the artificial dielectric. As we illustrate now, the UPEM can also provide an intuitive insight into those properties without resorting to extensive computation.

For that purpose, we consider the blazed area-coded effective-medium gratings shown in Fig. 1(b) (bottom). These gratings are composed of a periodic association of pie slices with a subwavelength transverse periodicity and have been recently fabricated with an electron-beam lithography tool in quartz for operation at $633 \mathrm{~nm} \mathrm{[16].}$ The reported experimental efficiencies $(\approx 70 \%)$ have been found to be in very good agreement with theoretical predictions. Using the UPEM, we aim at showing that, although they are apparently fully different, blazed areacoded effective-medium DOEs and 1D blazed-binary DOEs provide identical performances.

For that purpose, in Fig. 6(a), we show a top view of an area-coded grating with a period $L$ along the $x$ direction and with a subwavelength periodicity $\Lambda_{s}$ along the transverse $y$ direction. In Fig. 6(b), we consider a $1 \mathrm{D}$ blazedbinary grating with the same period $L$ and with a sampling period, defined as the separation distance between the subwavelength ridges, equal to the transverse periodicity $\Lambda_{s}$ of the area-coded element. Figures 6(c) and 6(d) show the associated local geometries. For both cases, the geometries are identical subwavelength lamellar gratings, which are rotated by $\pi / 2$ from each other. Thus, provided that the blazed-binary and the area-coded gratings are fabricated in the same material using the same calibration function $g=F(\psi)$, the associated local geometries of both gratings are strictly identical. In the limit of infi-

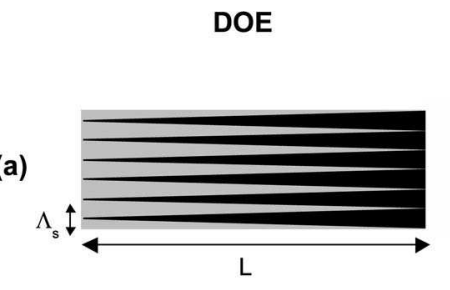

Local geometry (b)

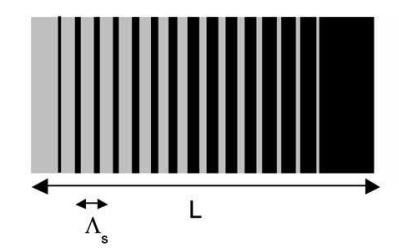

(e)

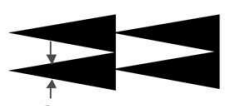

$\mathrm{f} \Lambda_{\mathrm{s}}$

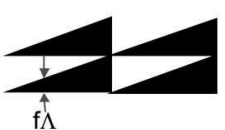

$\AA_{\mathrm{s}}^{4}$ (c)

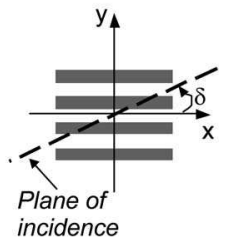

(d)
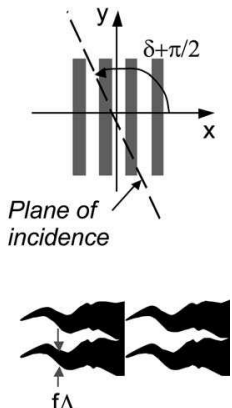

Fig. 6. Equivalence in the long-period limit between area-coded effective medium gratings and blazed-binary gratings composed of subwavelength ridges. (a), (c) Area-coded effective-medium grating and the associated local geometry. (b), (d) Blazed-binary grating with subwavelength ridges and the associated local geometry. Note that the subwavelength transverse period $\Lambda_{s}$ in (a) is equal to the sampling period of the blazed-binary element in (b). The local geometries in (c) and (d) are identical, except for a $\pi / 2$ rotation marked by the azimuthal angle $\delta$. (e) Three different area-coded gratings with identical optical properties in the long-period limit. Note that the local fraction of high-index material $f$ is the same in all figures.

nitely large periods, within the model framework based on the exploitation of the local transmittance, this implies that the optical properties of both gratings are strictly identical provided that the plane of incidence, defined by the azimuthal angle $\delta$ in Figs. 6(c) and 6(d), is rotated by $\pi / 2$. More specifically, the diffraction efficiencies in all orders, including the first order and the background orders as well, are the same for any polarization and for any angle of incidence. As illustrated in Fig. 6(e), this additionally implies that different area-coded gratings that apparently possess different geometries with an identical local fraction of dielectric material have identical optical responses.

Clearly these conclusions only hold in the limit of large periods, i.e., in the limit of slowly varying unwrapped phase functions. In the resonance domain and for periods that are not much larger than the wavelength of the incident illumination, the optical responses of all these gratings may largely differ from one to another. However, from the vector theory calculations reported in [15,34] for area-coded and blazed-binary gratings, similar optical responses with only few percent differences into the first diffracted order may be anticipated for zone widths as small as $10 \lambda$. It is therefore anticipated that even in the resonance domain, area-coded gratings, like blazedbinary gratings with subwavelength ridges, may suffer from form-birefringence effects that prevent the realization of efficient blazes for unpolarized applications $[11,22]$. This does not represent a limitation for blazedbinary DOEs composed of subwavelength centrosymmetric elements like pillars or holes [24,35]. 


\section{CONCLUSIONS}

We have presented a hybrid scalar-vector method for the analysis of DOEs with slowly varying phase functions. The model fully relies on the target unwrapped phase function that the optical designer needs to encode for correcting the aberrations of the optical system. The far-field DOE response is then represented as a coherent superposition of different elementary wave fields, each wave field corresponding to a diffraction order that is fully represented by the known unwrapped phase function. This superposition provides an intuitive and meaningful representation of the background noise, especially when only the few highest-efficiency background orders are considered. The method can be applied to various DOEs composed of different features, such as classical échelettes, or more complex patterns formed with subwavelength holes, grooves, or pie slices. For all of these cases, we showed that with moderate computational loads, the DOE optical response can be fully evaluated as a function of the incidence angle or of the illumination wavelength. Such analysis leads to the prediction of the energy diffracted into the targeted order and of the deterministic loss into all background diffraction orders. We believe that this new tool will be helpful for larger integration of DOEs in tomorrow's optical systems, as it provides accurate performance predictions that may be used for efficient design and optimization of a large variety of DOE structures.

\section{ACKNOWLEDGMENTS}

The authors thank Christophe Sauvan and Jean-Paul Hugonin for fruitful discussions. This work was supported by the French Délégation Générale de l'Armement under contract 0334011 . The European Network of Excellence NEMO is acknowledged for enabling additional informal exchanges between partners.

\section{REFERENCES}

1. M. Testorf, "Perturbation theory as a unified approach to describe diffractive optical elements," J. Opt. Soc. Am. A 16, 1115-1123 (1999).

2. J. Turunen, "Diffraction theory of microrelief gratings," in Micro-Optics, H. P. Herzig, ed. (Taylor \& Francis, 1997), pp 31-52.

3. A. V. Pfeil, F. Wyrowski, A. Drauschke, and H. Aagedal, "Analysis of optical elements with the local plane-interface approximation," Appl. Opt. 39, 3304-3313 (2000).

4. H. Lajunen, J. Tervo, J. Turunen, T. Vallius, and F. Wyrowski, "Simulation of light propagation by local spherical interface approximation," Appl. Opt. 42, 6804-6810 (2003).

5. J. M. Finlan, K. M. Flood, and R. J. Bojko, "Efficient f/1 binary-optics microlenses in fused silica designed using vector diffraction theory," Opt. Eng. 34, 3560-3564 (1995).

6. E. Noponen, J. Turunen, and A. Vasara, "Parametric optimization of multilevel diffractive optical elements by electromagnetic theory,” Appl. Opt. 31, 5910-5912 (1992).

7. C. Sauvan, P. Lalanne, and M. S. L. Lee, "Broadband blazing with artificial dielectrics," Opt. Lett. 29, 1593-1595 (2004).

8. H. Lajunen, J. Turunen, and J. Tervo, "Design of polarization gratings for broadband illumination," Opt. Express 13, 3055-3067 (2005).

9. M. S. L. Lee, P. Lalanne, J. C. Rodier, P. Chavel, E. Cambril, and Y. Chen, "Imaging with blazed-binary diffractive elements," J. Opt. A, Pure Appl. Opt. 4, 119-124 (2002).

10. P. Lalanne, S. Astilean, P. Chavel, E. Cambril, and H. Launois, "Design and fabrication of blazed-binary diffractive elements with sampling periods smaller than the structural cutoff," J. Opt. Soc. Am. A 16, 1143-1156 (1999).

11. J. Tervo and J. Turunen, "Paraxial-domain diffractive elements with $100 \%$ efficiency based on polarization gratings," Opt. Lett. 25, 785-787 (2000).

12. J. Tervo, V. Kettunen, M. Honkanen, and J. Turunen, "Design of space-variant diffractive polarization elements," J. Opt. Soc. Am. A 20, 282-289 (2003).

13. E. Hasman, V. Kleiner, G. Biener, and A. Niv, "Polarization dependent focusing lens by use of quantized Pancharatnam-Berry phase diffractive optics," Appl. Phys. Lett. 82, 328-360 (2003).

14. B. H. Kleemann, J. Ruoff, and R. Arnold, "Area coded effective medium (ACE) structures, a new type of grating design," Opt. Lett. 30, 1617-1619 (2005).

15. U. Levy, H. C. Kim, C. H. Tsai, and Y. Fainman, "Nearinfrared demonstration of computer-generated holograms implemented by using subwavelength gratings with spacevariant orientation," Opt. Lett. 30, 2089-2091 (2005).

16. H. Elfström, M. Kuittinen, T. Vallius, B. H. Kleemann, J. Ruoff, and R. Arnold, "Fabrication of blazed gratings by area-coded effective medium structures," Opt. Commun. 266, 697-703 (2006).

17. B. Layet and M. Taghizadeh, "Analysis of gratings with large periods and small feature sizes by stitching of the electromagnetic field," Opt. Lett. 21, 1508-1510 (1996).

18. T. R. M. Sales and G. M. Morris, "Diffractive-refractive behavior of kinoform lenses," Appl. Opt. 36, 253-257 (1997).

19. O. Sandfuchs, R. Brunner, D. Pätz, S. Sinzinger, and J. Ruoff, "Rigorous analysis of shadowing effects in blazed transmission gratings," Opt. Lett. 31, 3638-3640 (2006).

20. J. C. Jonsson and F. Nikolajeff, "Optical properties of injection molded subwavelength gratings," Opt. Express 12, 1924-1931 (2004).

21. H. Haidner, J. T. Sheridan, J. Schwider, and N. Streibl, "Design of a blazed grating consisting of metallic subwavelength binary grooves," Opt. Commun. 98, 5-10 (1993).

22. J. R. Wendt, G. A. Vawter, R. E. Smith, and M. E. Warren, "Subwavelength, binary lenses at infrared wavelengths," J. Vac. Sci. Technol. B 15, 2946-2949 (1997).

23. S. Astilean, P. Lalanne, P. Chavel, E. Cambril, and H. Launois, "High efficiency subwavelength diffractive element patterned in a high-refractive-index material for 633 nm," Opt. Lett. 23, 552-554 (1998).

24. F. T. Chen and H. G. Craighead, "Diffractive lens fabricated with mostly zeroth-order gratings," Opt. Lett. 21, 177-179 (1996).

25. G. J. Swanson, "Binary optics technology: the theory and design of multilevel diffractive optical elements," MIT Technical Report 854 (MIT, 1989).

26. H. Dammann, "Phase holograms of diffuse objects," J. Opt. Soc. Am. A 60, 1635-1639 (1970).

27. D. A. Buralli and G. M. Morris, "Effect of diffraction efficiency on the modulation transfer function of diffractive lenses," Appl. Opt. 31, 4389-4396 (1992).

28. J.-P. Hugonin and P. Lalanne, "Reticolo software for grating analysis," trademark of the Institut d'Optique (2005).

29. M. G. Moharam, E. B. Grann, D. A. Pommet, and T. K. Gaylord, "Formulation for stable and efficient implementation of the rigorous coupled-wave analysis of binary gratings," J. Opt. Soc. Am. A 12, 1068-1076 (1995).

30. P. Lalanne and G. M. Morris, "Highly improved convergence of the coupled-wave method for TM polarization,” J. Opt. Soc. Am. A 13, 779-784 (1996).

31. G. Granet and B. Guizal, "Efficient implementation of the coupled-wave method for metallic lamellar gratings in TM polarization," J. Opt. Soc. Am. A 13, 1019-1023 (1996)

32. L. Li, "New formulation of the Fourier modal method for 
crossed surface-relief gratings," J. Opt. Soc. Am. A 14, 2758-2767 (1997).

33. W. H. Press, B. P. Flannery, S. A. Teukolsky, and W. T. Vetterling, Numerical Recipes: The Art of Scientific Computing (Cambridge U. Press, 1989), Chap. 3.

34. M. S. L. Lee, P. Lalanne, and P. Chavel, "Blazed-binary diffractive elements with periods much larger than the wavelength," J. Opt. Soc. Am. A 17, 1250-1255 (2000).

35. P. Lalanne, S. Astilean, P. Chavel, E. Cambril, and H. Launois, "Blazed-binary subwavelength gratings with efficiencies larger than those of conventional échelette gratings," Opt. Lett. 23, 1081-1083 (1998). 